\title{
Dynamics of a turbulent layer generated by velocity jump
}

\author{
D. V. Strunin* A. J. Roberts ${ }^{\dagger}$
}

(Received 14 January 2003)

\begin{abstract}
We study a growing turbulent layer generated by the shear between half-spaces of fluid moving with different velocities. The $K-\ell$ Kolmogorov theory of turbulence is used, with the closure relation being proportionality of turbulent spatial scale to layer thickness. We show that the turbulent layer exhibits complete self-similarity, that is self-similar dynamics that are independent of initial conditions. A criterion of stability of self-similar regimes is deduced in a simple analytical form. The value of a coefficient entering the closure relation is estimated by matching experimental data.
\end{abstract}

*Dept. Maths \& Computing, University of Southern Queensland, Toowoomba, AustraliA. mailto:strunin@usq.edu.au

$\dagger^{\dagger}$ Dept. Maths \& Computing, University of Southern Queensland, Toowoomba, Australia. mailto:aroberts@usq.edu.au

${ }^{0}$ See http: //anziamj . austms . org.au/V44/CTAC2001/Stru for this article,

(C) Austral. Mathematical Soc. 2003. Published 1 April 2003. ISSN 1446-8735 


\section{Contents}

1 Introduction

C724

2 Evolution of turbulent layer

C727

3 Stability of self-similar regimes

C731

4 Conclusions

C736

References

C736

\section{Introduction}

Turbulence between parallel flows of fluid moving with different velocities $U_{1}$ and $U_{2}$ presents a classical example of shear turbulence. We consider the situation where each flow extends over a half-space and velocity distribution is uniform in each flow. The dynamics are described by the Kolmogorov phenomenological theory of fully developed turbulence [6]. Over the decades this theory has provided the foundation for numerous applications of so-called $K-\ell$ model of turbulence. For horizontally statistically uniform turbulent layer this model writes [9]:

$$
\partial_{t} K=\alpha \partial_{z}\left(\ell \sqrt{K} \partial_{z} K\right)-c \frac{K^{3 / 2}}{\ell}+\ell \sqrt{K}\left(\partial_{z} u\right)^{2}, \partial_{t} u=\partial_{z}\left(\ell \sqrt{K} \partial_{z} u\right),
$$

where $K$ is the turbulent energy, that is the ensemble-average kinetic energy of pulsations per unit mass; $z$ is the transverse coordinate; $t$ is the time; $\alpha$ and $c$ are positive coefficients; $u$ is the average velocity; and $\ell$ is the spatial scale of turbulence. The value $\ell$ is implied to obey either its own transfer equation or some simpler relation which 
closes the problem. The first equation (1) describes the evolution of the turbulent energy due to the transfer by velocity pulsations (nonlinear diffusion term), viscous dissipation (negative term) and production by the shear; the second equation (1) describes the turbulent diffusion of the momentum.

The scale $\ell$ characterizes the typical size of turbulent eddies. We choose to adhere a simple closure approximation [1]: the size equals some fixed portion of the thickness of the layer, which we denote by $2 h(t)$. Thus,

$$
\ell(t)=\mu h(t) .
$$

The parameter $\mu$ will be determined by matching the growth rate of the turbulent layer to the experimental data.

Theoretically it has long been established that the thickness of the layer increases linearly in time: $h \sim t$. It is commonly assumed that this law trivially follows from the dimensional consideration that the thickness depends on the initial velocity jump, $2 U$, and time, leading to

$$
h=\lambda U t,
$$

where $\lambda$ is a non-dimensional coefficient.

However, generally the dynamics also depend on initial conditions such as the initial thickness of the layer, $a$, and the initial amount of the turbulent energy, $Q$, emerging as a result of the initial instability of the laminar flow (this stage is not covered by the $K-\ell$ model). The presented dimensional analysis implicitly suggests that at large times the role of the parameters $a$ and $Q$ vanishes. However, whether or not this occurs is not straightforward. The book of Barenblatt [2] contains many examples where small (dimensionless) parameters remain important because solutions have power law asymptotics; for example

$$
F(\xi, \eta) \sim \eta^{\lambda} G\left(\xi / \eta^{\nu}\right) \quad \text { as } \eta \rightarrow 0 .
$$


Imagine that $\eta$ here stands for the dimensionless combination of variables $a /(U t) \rightarrow 0$ as $t \rightarrow \infty$. If (4) holds the parameter $a$ cannot be ignored at all times. Such a situation is referred to as incomplete self-similarity on the parameter $\eta$. This is distinguished from complete self-similarity when

$$
F(\xi, \eta) \sim F(\xi, 0) \quad \text { as } \eta \rightarrow 0,
$$

where $F(\xi, 0)$ is non-zero and finite. We will see that the expansion law (3) might be a part of solution representing either complete or incomplete self-similarity. In the former case the role of $a$ and $Q$ would vanish, but in the latter case these parameters always remain important. Which type of self-similarity actually takes place presents special interest.

Kasyanova [5] used the model (1-2) to study a problem of the shear flow in the upper layer of the ocean. They suggested that the flow was driven by a constant stress on the surface. This situation is different from our case which is equivalent to the dynamics of a half-space of fluid with prescribed constant velocity on the boundary.

Experimental data on the shear turbulence between plane mixing layers are presented and discussed in [3, 10, 7, 8, 11, 15]. Experimentalists used statistically stationary flows of gas having different velocities, separated by a barrier. The flows come into contact at a barrier's end, and the mixing zone gradually widens downstream. This process is similar to the mixing of half-spaces of fluid, impulsively set into motion at the initial moment with different velocities. To transform the spatial problem into the temporal problem the time in (3) was linked [3] to the downstream coordinate $x$ as $t=x / U_{c}$, where $U_{c}$ is some effective (convective) velocity. With $U_{c}$ assumed equal to the average velocity, $U_{c}=\left(U_{1}+U_{2}\right) / 2$, relation (3) becomes $2 h / x=2 \lambda\left(U_{1}-U_{2}\right) /\left(U_{1}+U_{2}\right)$. This formula was used in [3] 
to process the measurements, to yield the estimate

$$
\lambda=0.1 \text {. }
$$

\section{Evolution of turbulent layer}

We study the system (1-2) with the boundary conditions of uniform flow and zero turbulence far away from the growing turbulent layer:

$$
K( \pm \infty, t)=0, \quad u( \pm \infty, t)= \pm U .
$$

The coefficients $\alpha$ and $c$ are assumed to be constants: $\alpha=1$, $c=0.062[9,4]$. Given the conditions (6) and for a wide range of assumptions about $\ell$, the nonlinear turbulent diffusion mechanism leads to the turbulent energy being confined within a layer of finite width $2 h(t)$. Beyond the layer the functions $K(z, t)$ and $u(z, t)$ remain unperturbed. Thus the eddy size $\ell$ appears to be well defined in (2).

We assume that the velocity profile is anti-symmetric with respect to the middle plane, $z=0$, and the turbulent energy profile is symmetric with respect to this plane. Therefore it suffices to study only half of the layer, say $z>0$, under additional boundary conditions expressing zero energy flux and zero velocity on the middle plane:

$$
\left.\partial_{z} K(z, t)\right|_{z=0}=0, \quad u(0, t)=0 .
$$

As for the initial conditions, we adopt arbitrary initial profiles of the velocity, $u(z, 0)$, and turbulent energy $K(z, 0)$ in an initial layer of width $2 a$. Thus there is some initial charge of the turbulent energy:

$$
Q=\int_{-a}^{a} K(z, 0) d z .
$$


The turbulent dynamics are determined by the dimensional parameters

$$
U(\mathrm{~cm} / \mathrm{s}), \quad a(\mathrm{~cm}), \quad Q\left(\mathrm{~cm}^{3} / \mathrm{s}^{2}\right), \quad z(\mathrm{~cm}), \quad t(\mathrm{~s}) .
$$

Two of these are dimensionally independent: let us choose $U$ and $t$. The unknowns to be determined are

$$
K\left(\mathrm{~cm}^{2} / \mathrm{s}^{2}\right), \quad u(\mathrm{~cm} / \mathrm{s}), \quad h(\mathrm{~cm}) .
$$

Non-dimensionalizing every quantity by the appropriate combination of $U$ and $t$ we get:

$$
\begin{gathered}
K=U^{2} R\left(\frac{z}{U t}, \frac{a}{U t}, \frac{Q}{U^{3} t}\right), \\
u=U V\left(\frac{z}{U t}, \frac{a}{U t}, \frac{Q}{U^{3} t}\right), \\
h=U t H\left(\frac{a}{U t}, \frac{Q}{U^{3} t}\right),
\end{gathered}
$$

where $R, V$ and $H$ are dimensionless functions. We will use these expressions to analyze the numerical results.

In the computations we transform to non-dimensional variables:

$$
h=a h_{1}, \quad z=a z_{1}, \quad t=(a / U) t_{1}, \quad u=U u_{1}, \quad K=U^{2} K_{1} .
$$

In non-dimensional form, equations (1) are written as (henceforth the subscripts are omitted unless specified otherwise),

$$
\begin{aligned}
& \partial_{t} K=\alpha \mu h \partial_{z}\left(\sqrt{K} \partial_{z} K\right)-c \frac{K^{3 / 2}}{\mu h}+\mu h \sqrt{K}\left(\partial_{z} u\right)^{2}, \\
& \partial_{t} u=\mu h \partial_{z}\left(\sqrt{K} \partial_{z} u\right) \\
& K(\infty, t)=0, \quad u(\infty, t)=1 .
\end{aligned}
$$




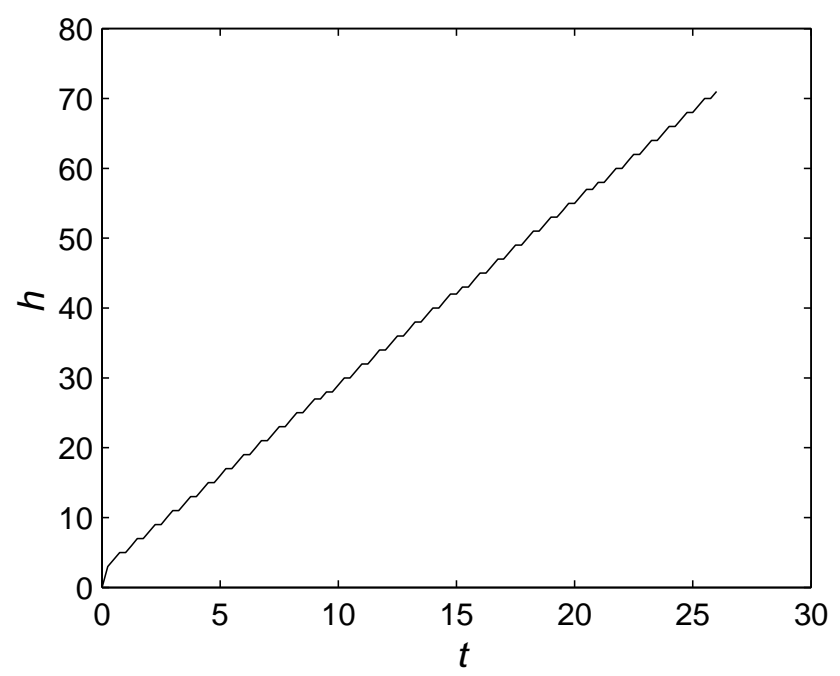

FiguRE 1: The turbulent layer grows linearly in time: $\mu=1$.

The initial conditions have the form

$$
\begin{aligned}
& K=K_{0}(z, 0) \quad \text { and } \quad u=u_{0}(z, 0) \quad \text { for } 0<z<1 \\
& K \equiv 0 \quad \text { and } \quad u \equiv 1 \quad \text { for } z \geq 1
\end{aligned}
$$

where $K_{0}$ and $u_{0}$ are some arbitrary functions.

We solved the problem expressed in equations (12-16) using the Crank-Nicolson finite difference scheme. Discretized equations were solved with the help of the IMSL Fortran library subroutine. As in [12], we slightly modified the initial condition by assuming that outside of the turbulent layer the turbulent energy equals some small value $\varepsilon$. We checked that the dynamics were not sensitive to the specific value of $\varepsilon$, provided $\varepsilon$ was sufficiently small. Thus, our solutions effectively correspond to the limit $\varepsilon \rightarrow 0$.

Figures 1-3 display the solutions for half of the layer, $z>0$. The picture for the whole layer is obtained by extending the tur- 

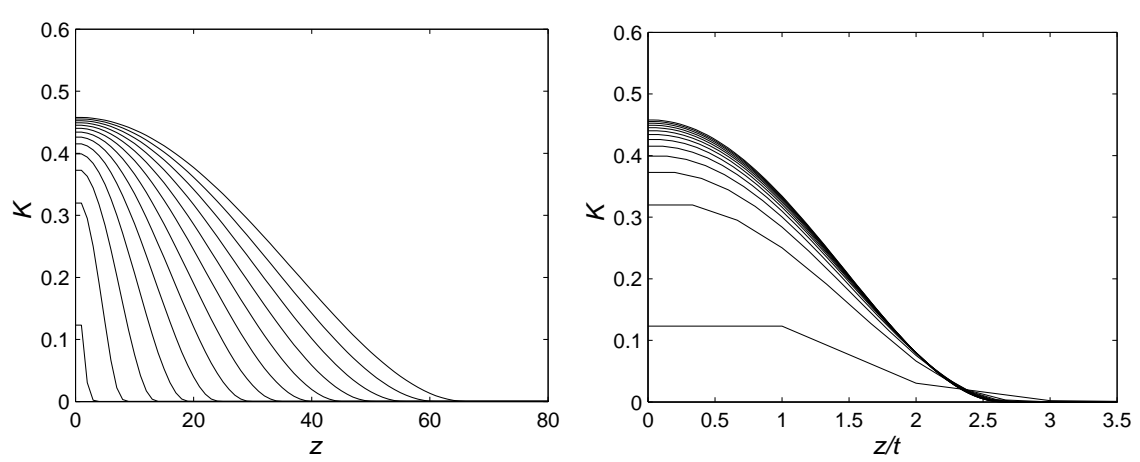

Figure 2: The turbulent energy approaches self-similar regime: $\mu=1 ; t=6,15, \ldots, 150$ with interval 15 .
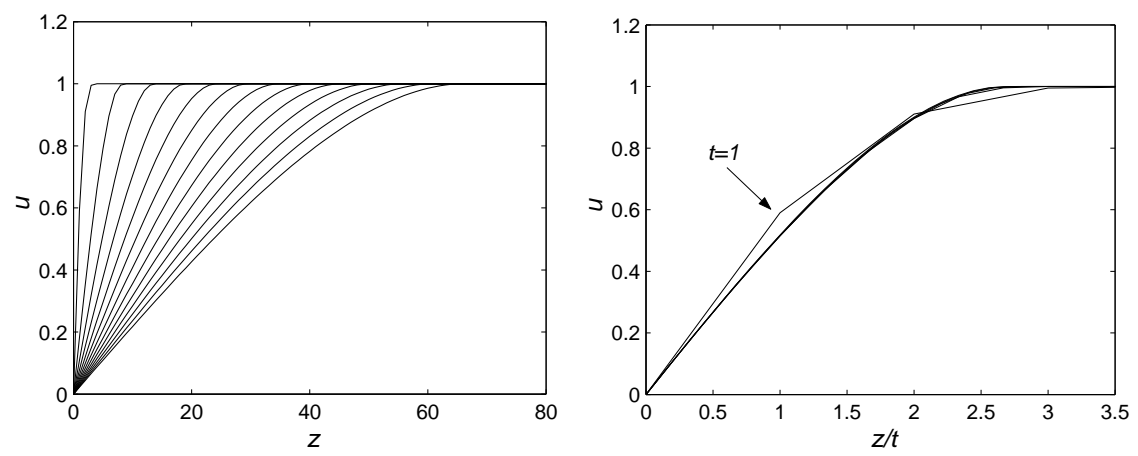

FiguRE 3: The velocity approaches self-similarity regime: $\mu=1$; $t=15, \ldots, 150$ with interval 15 . 
bulent energy profile symmetrically and the velocity profile antisymmetrically into the region $z<0$. The figures demonstrate that the asymptotic solutions at large times become self-similar. The thickness of the turbulent layer versus time very quickly approaches a straight line, $h \sim t$. According to (11) this means that the function $H\left[a /(U t), Q /\left(U^{3} t\right)\right]$ tends to a finite limit. However, this alone does not guarantee that the parameters $a /(U t)$ and $Q /\left(U^{3} t\right)$ can be replaced by 0 , because the following asymptotic form of the function $H$ is possible

$$
H\left(\frac{a}{U t}, \frac{Q}{U^{3} t}\right)=H\left[\frac{a /(U t)}{Q /\left(U^{3} t\right)}\right]=H\left(\frac{a U^{2}}{Q}\right) .
$$

If valid, this hypothetical form of $H$ contains no time so that $H$ is finite as $t \rightarrow \infty$. If valid this expression would represent incomplete self-similarity with respect to any of the parameters $Q /\left(U^{3} t\right)$ and $a /(U t)$ (which corresponds to $\lambda=0, \nu=1$ in the definition of incomplete similarity (4)). We conducted a number of numerical experiments to determine whether or not the solution is sensitive to $a U^{2} / Q$. No dependence on this combination is found. Thus, the function $H$ not only has finite non-zero limit as $a /(U t) \rightarrow 0$ and $Q /\left(U^{3} t\right) \rightarrow 0$, but, in addition, this limit is independent of $a U^{2} / Q$. Analogously the variables $a /(U t)$ and $Q /\left(U^{3} t\right)$ may be replaced by 0 in (9) and (10).

\section{$3 \quad$ Stability of self-similar regimes}

The numerical results demonstrate both existence and stability of the self-similar regimes. At the same time it is possible to derive a general criterion of stability in useful analytical form. To simplify manipulations, the parameter $\mu$ is removed from the problem formulations, by transferring to a new set of non-dimensional variables 
(labelled by subscripts):

$$
z=\mu z_{2}, \quad h=\mu h_{2}, \quad c=\mu^{2} c_{2} .
$$

Using (17), the non-dimensional governing equations and boundary conditions assume the following form, with the subscripts being omitted:

$$
\begin{aligned}
& \partial_{t} K=\alpha h \partial_{z}\left(\sqrt{K} \partial_{z} K\right)-c \frac{K^{3 / 2}}{h}+h \sqrt{K}\left(\partial_{z} u\right)^{2}, \\
& \partial_{t} u=h \partial_{z}\left(\sqrt{K} \partial_{z} u\right), \\
& K=0 \text { and } u= \pm 1 \quad \text { on } z= \pm h(t) .
\end{aligned}
$$

Denoting $\sqrt{K}=\beta$, transform to similarity variables

$$
\eta=z / t, \quad h=M t
$$

and make the log-time transformation [14]:

$$
\tau=\log t .
$$

The log-time transformation proved useful in stability analysis of the processes involving usual Fickian diffusion [13]. The transformation convert the diffusion equations to a form where small perturbations of large physical size decay exponentially in the logarithmic time $\tau$, in contrast to algebraic decay when expressed in terms of the usual time $t$. That the Wayne transformation should work for the turbulent layer is conjectured by the following analogy with the Fickian diffusion. For the latter process the layer thickness $h$ would be proportional to $\sqrt{t}$ and hence, with a constant diffusion coefficient $D$, the mixing time across the layer is $h^{2} / D \sim t$. The thickness of the turbulent layer increases linearly in time, $h \sim t$, but the diffusion coefficient $h \sqrt{K}$ also increases linearly in time as $K$ is constant. Thus the mixing time across the turbulent layer is similarly $h^{2} /(h \sqrt{K}) \sim t$. Hence, the qualitative property of algebraic decay 
of perturbations in the Fickian diffusion should also take place for the turbulent layer. Using (21-22) we calculate the derivatives

$$
\partial_{z}=\frac{1}{t} \partial_{\eta}, \quad \partial_{t}=\frac{1}{t}\left(\partial_{\tau}-\eta \partial_{\eta}\right) .
$$

Substituting these into (18-20) we obtain

$$
\begin{aligned}
\beta_{\tau}-\eta \beta_{\eta}= & \alpha M\left(\beta \beta_{\eta \eta}+2 \beta_{\eta}^{2}\right)-\frac{c}{2 M} \beta^{2}+\frac{M}{2}\left(u_{\eta}\right)^{2}, \\
u_{\tau}-\eta u_{\eta}= & M\left(\beta u_{\eta}\right)_{\eta} \\
\beta=0 & \text { and } \quad u= \pm 1 \text { for } \eta= \pm M
\end{aligned}
$$

To linearize the problem, the self-similar solution is perturbed,

$$
\beta(\eta, \tau)=\beta(\eta)+\beta^{\prime}(\eta) e^{\lambda \tau}, \quad u(\eta, \tau)=u(\eta)+u^{\prime}(\eta) e^{\lambda \tau},
$$

where the primes denote the small perturbations. Substituting (26) into (23-24) and neglecting products of perturbation quantities we deduce

$$
\begin{aligned}
\lambda \beta^{\prime}= & \eta \beta_{\eta}^{\prime}+\alpha M\left(\beta \beta_{\eta \eta}^{\prime}+\beta_{\eta \eta} \beta^{\prime}+4 \beta_{\eta} \beta_{\eta}^{\prime}\right) \\
& -\frac{c}{M} \beta \beta^{\prime}+M u_{\eta} u_{\eta}^{\prime}, \\
\lambda u^{\prime}= & \eta u_{\eta}^{\prime}+M\left(u_{\eta} \beta^{\prime}+\beta u_{\eta}^{\prime}\right)_{\eta} .
\end{aligned}
$$

Now multiply (27) by $\beta^{\prime}$ and (28) by $u^{\prime}$, sum up the equations and integrate over the infinite domain. Integration by parts gives

$$
\begin{aligned}
& \lambda \int_{-\infty}^{\infty}\left[{\beta^{\prime}}^{2}+u^{\prime 2}\right] d \eta=\left[\frac{1}{2} \eta u^{\prime 2}+M u_{\eta} \beta^{\prime} u^{\prime}+M \beta u^{\prime} u_{\eta}^{\prime}+\frac{1}{2} \eta \beta^{\prime 2}\right. \\
& \left.+\alpha M\left(\beta \beta^{\prime} \beta_{\eta}^{\prime}+\frac{3}{2} \beta_{\eta}{\beta^{\prime}}^{2}\right)\right]_{-\infty}^{\infty}-\int_{-\infty}^{\infty}\left[\frac{1}{2}{\beta^{\prime}}^{2}+\alpha M \beta{\beta^{\prime}}_{\eta}^{2}\right. \\
& \left.+\frac{c}{M}{\beta^{\prime 2}}^{2}+\frac{1}{2} u^{\prime 2}+M \beta{u^{\prime}}_{\eta}^{2}+\frac{1}{2} \alpha M{\beta^{\prime}}^{2} \beta_{\eta \eta}\right] d \eta
\end{aligned}
$$


According to (25), $\beta, \beta_{\eta}$ and $u_{\eta}$ vanish outside of the turbulent layer, so the terms involving these quantities at infinity disappear. Thus we only need to assume that $u^{\prime}$ and $\beta^{\prime}$ are $o(1 / \sqrt{\eta})$ for large $\eta$ for the other two boundary terms to disappear. The sufficient condition for $\lambda$ to be negative is then

$$
\frac{1}{2}\left(1+\alpha M \beta_{\eta \eta}\right)+\frac{c}{M} \beta \geq 0 \text { for all } \eta .
$$

This condition ensures that the self-similar solution is asymptotically stable to small perturbations, and hence is a realised attractor.

Interestingly, there exists a set of the values of $c$ and $\alpha$ leading to exact analytic solutions. For such a solution we can easily check whether the criterion (29) holds. Denote the limit of the function $H$ in (11) as $\xi_{0}$ and define the self-similar variable as

$$
\xi=\frac{z}{U t} \text {. }
$$

Thus, $\xi$ varies in the interval $0 \leq \xi \leq \xi_{0}$. Note that the variables $\eta$, $M$ and $\xi, \xi_{0}$ are connected:

$$
\eta=\xi / \mu, \quad M=\xi_{0} / \mu .
$$

As established numerically the functions $H, R$ and $V$ have finite non-zero limits:

$$
H=\xi_{0}, \quad R=R(\xi), \quad V=V(\xi) .
$$

Substituting (9-11) and (30-32) into (1) we obtain ordinary differential equations for $R$ and $V$ :

$$
\begin{aligned}
& \xi \frac{d R}{d \xi}+\alpha \mu \xi_{0} \frac{d}{d \xi}\left(R^{1 / 2} \frac{d R}{d \xi}\right) \\
& -\frac{c}{\mu \xi_{0}} R^{3 / 2}+\mu \xi_{0} R^{1 / 2}\left(\frac{d V}{d \xi}\right)^{2}=0 \\
& \xi \frac{d V}{d \xi}+\mu \xi_{0} \frac{d}{d \xi}\left(R^{1 / 2} \frac{d V}{d \xi}\right)=0
\end{aligned}
$$




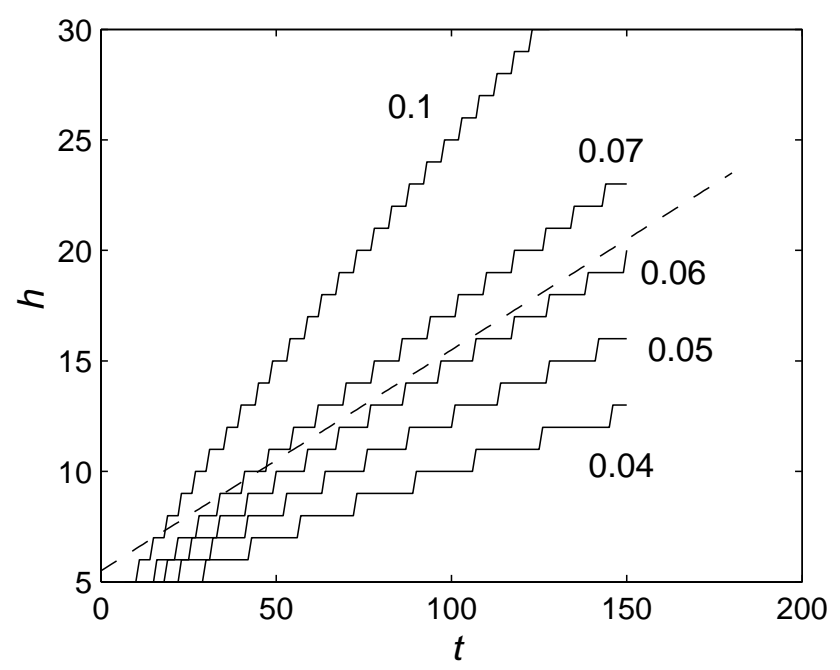

Figure 4: Experimental (dashed line) and theoretical growth of the turbulent layer for various $\mu$.

It is easy to verify that for $c=0$ and $\alpha=2 / 5$ the analytic solution of $(33-34)$ is

$$
\xi_{0}=\mu \sqrt{5 / 2}, \quad R^{1 / 2}=\frac{\sqrt{5 / 2}}{2}-\frac{\xi^{2}}{\sqrt{10} \mu^{2}}, \quad V=\frac{\sqrt{2 / 5} \xi}{\mu} .
$$

Now, using (35) and (31) we find:

$$
\frac{1}{2}\left(1+\alpha M \beta_{\eta \eta}\right)+\frac{c}{M} \beta=\frac{1}{2}\left[1+\alpha \frac{\xi_{0}}{\mu} \frac{d^{2} R^{1 / 2}}{d(\xi / \mu)^{2}}\right]=\frac{3}{10}>0 ;
$$

therefore the regime (35) is stable. Although the exact solution (35) is not directly useful because the value $\alpha=2 / 5$ is too small, it may act as a useful reference point for further analysis.

Finally we determined the value of the parameter $\mu$ for which theoretical growth rate matches experiments (Figure 4). According to (5), the experimental line in Figure 4 is drawn with the 
slope $\lambda=0.1$. The step-like form of the theoretical curves here is due to discretization: the turbulent front moves by jumps over grid elements. This is just an approximation of real continuous propagation of the front. See that the best match is achieved for $\mu=0.06$.

\section{Conclusions}

A version of the $K-\ell$ model of fully developed turbulence is used to analyze the expansion of a turbulent layer between parallel fluid flows initially moving with different constant velocities. It is numerically demonstrated that large-time asymptotic solutions are independent of initial conditions and thus are examples of complete selfsimilarity. In the limit $t \rightarrow \infty$ turbulence tends to occupy an entire space, with an intensity of velocity pulsations approaching a finite limit at every point of space. A criterion of stability of self-similar regimes is deduced in analytical form. The value of a coefficient entering the closure relation of the $K-\ell$ model is estimated using available experimental data.

Acknowledgements: The authors thank the Australian Research Council for their support of this research.

\section{References}

[1] G. I. Barenblatt, N. L. Galerkina and M. V. Luneva.

Evolution of a turbulent burst. J. Engin. Phys., 53:1246-1252, 1987. C725 
[2] G. I. Barenblatt. Scaling, Self-similarity, and Intermediate Asymptotics. Cambridge University Press, 1996. C725

[3] G. L. Brown and A. Roshko. On density effects and large structure in turbulent mixing layers. J. Fluid Mech., 64:775-816, 1974. C726

[4] W. Frost and T. H. Moulden (eds.) Handbook of Turbulence. Fundamentals and Applications. Plenum, New-York, 1977. $\mathrm{C} 727$

[5] N. Kasyanova. A numerical study of stabilization of a turbulent layer. MSc thesis, Moscow Institute of Physics and Technology, 1983. C726

[6] A. N. Kolmogorov. Equations of turbulent motion of incompressible fluid. Izv. Akad. Nauk SSSR, Ser. Fiz., 6:56-58, 1942. C724

[7] J. B. Miles and J. Shih. Similarity parameter for two-stream turbulent jet-mixing region. AIAA J., 6:1429, 1968. C726

[8] R. D. Mills. Numerical and experimental investigations of the shear layer between two parallel streams. J. Fluid Mech., 33:591, 1968. C726

[9] A. S. Monin and A. M. Yaglom. Statistical Fluid Mechanics. Mechanics of Turbulence Vol.1. MIT Press, Cambridge-London, 1971. C724, C727

[10] A. Roshko. Structure of turbulent shear flows: a new look. AIAA J., 14:1349-1357, 1976. C726

[11] H. Schlichting. Boundary Layer Theory. McGraw-Hill, 1960. C726 
[12] D. V. Strunin. Transition to self-similarity of diffusion of tracer in turbulent patch. J. Engin. Mech., to appear in 127, No. 11, 2001. C729

[13] S. A. Suslov and A. J. Roberts. Similarity, attraction and initial conditions in an example of nonlinear diffusion. J. Austral. Math. Soc. B, 40(E):E1-E26, 1998. C732

[14] C. E. Wayne. Invariant manifolds for parabolic partial differential equations on unbounded domains. Arch. Rat. Mech. Anal., to appear. C732

[15] I. Wygnanski and H. E. Fiedler. The two-dimensional mixing region. J. Fluid Mech., 41:327, 1970. C726 